\title{
Identification, Screening and Characterization of Potential Probiotics from Human Milk
}

Marwah Ali Oudah ${ }^{1}$, K. Rajyalakshmi ${ }^{2}$, A. Shanti Priya ${ }^{3}$, Syed Shabana ${ }^{4}$, A. Krishna Satya ${ }^{5 *}$

1- Department of Biotechnology, Acharya Nagarjuna University, Nagarjuna Nagar, Guntur, Andhra Pradesh, India, Mobile no- 7842301514, Email: jaleelabdjaleel@gmail.com

2- Department of Biotechnology, Acharya Nagarjuna University, Nagarjuna Nagar, Guntur,

Andhra Pradesh, India, Mobile no- 9989723309, rajyalaxmik07@gmail.com

3- Sri Venkateswara College of Pharmacy, Madhapur, Hyderabad, Mobile no- 9849672429, Email: santhipriya2011@gmail.com

4- Department of Biotechnology, Acharya Nagarjuna University, Nagarjuna Nagar, Guntur,

Andhra Pradesh, India, Mobile no- 9381407027, syedshabana913@gmail.com

5, * - Department of Biotechnology, Acharya Nagarjuna University, Nagarjuna Nagar, Guntur, Andhra Pradesh, India, Mobile no- 9490639577, Email: akrishnasatya78@gmail.com

\section{ABSTRACT}

Several studies have demonstrated that breast milk is the main source of lactic acid bacteria which is a potential probioticwith nutritional and immunological function. Probiotics are the live microorganisms which when administrated as food supports human health. The main objective in the present investigation is isolation of potential probiotic lactobacillus species and identification based on morphological and biochemical characterization.By using Polymerase chain reaction (PCR) with primer pair of $16 \mathrm{~S}$ rRNA based molecular characterization specific to Lactobacillus species molecular identification was evaluated. Lactobacillus species were subjected to probioticand bio-preservative tests which include acid tolerance $(\mathrm{pH} 2,3,4,5,6,7)$, bile tolerance $(0.3,0.5,1 \%)$, phenol $(0.2,0.4 \%)$ and salt resistance $(3,6,9,12 \%)$. They are also tested for antibiotic, antimicrobial, hydrophobicity and auto aggregation abilities. MPS-16, MPS-9, MPS- 18, MPS-27 isolates showed best resistance to tetracycline, streptomycin, ampicillin, kanamycin, penicillin, vancomycin, ciprofloxacin antibiotics, and these isolates have the most survival ability under gastrointestinal conditions. Based on our work we can suggest that bacteria isolated from human breast milk islactic acid probiotic strain which can be used for further research and product development.

Keywords: Lactobacillus, Human milk, Potential Probiotic, Molecular Characterization, Antagonistic activity.

\section{Corresponding author: Dr. Alapati Krishna Satya}

\section{INTRODUCTION}

Probiotics are the live microorganisms which when administrated gives health benefits to the host (1) regulates the immune system, balance the healthy gut microbiome, protects from harmful pathogens, forbid the diarrhoea and constipation problem and lowers the cholesterol level and hypertension (2,3). Now a day's probiotics such as Lactobacillus, Streptococcus, Bifidobacterium and strains of yeast are generally used $(4,5)$ and these bacteria are treated as GRAS- Generally Recognised as Safe organisms by FDA (US food and drug administration) (6). Survival of bacteria under low $\mathrm{pH}$ (acidic condition) and high bile salt tolerance at GIT 
condition are the main criteria for probiotic to recommend for the host $(7,8)$. Until now the probiotic lactic acid bacteria were isolated from milk samples, dairy products, and different sources. The present investigation suggests that probiotic bacteria isolated from mother milk reduce the growth of the infectious diseases and increase the growth and development of body metabolic functions and immune system. $(9,10,11)$.

\section{METHODOLOGY:}

\section{Design:}

Human milk samples were collected from 10 healthy women between January 2018 and April 2018. Samples were collected from Government hospital and NRI hospital in Mangalagiri and oral informed consent was done from each individual. All the procedure was performed after affiliation by the Hospital heads and University permissions.

\section{Milk Sampling and Collection:}

Initially before sample collection breast skin was cleaned with Hand EX HC (chlorhexidine gluconate 2.0\%) and samples were collected three times for each woman (1) 30 min postpartum (before feeding) (2) 4-8 day post-partum and last (3) 25-30 day's post-partum. Sample was obtained from healthy women following the criteria include; type of delivery (vaginal delivery or cesarean section), Maternal body index- BMI (underweight, normal or overweight), Breast Skin alterations (allergies and dermatitis), drug usage, age complexion and number of pregnancies and abortion details (if any). Samples were collected under aseptic conditions and stored in the icebox until processing

\section{Isolation and Identification:}

MRS (de Man, Rogosa and Sharpe) agar medium has opted for the isolation of lactic acid bacteria. $1 \mathrm{ml}$ of human milk sample was added to $9 \mathrm{ml}$ of saline solution $(0.9 \%)$ and homogenized using vortex. The sample was serially diluted up to $10^{6}$ dilutions. $0.1 \mathrm{ml}$ of serially diluted sample was plated onto the MRS medium. At $37^{\circ} \mathrm{C}$ for $24-48$ hours plates were incubated. Clear colonies were selected and repeated morphology was shortlisted. Selected colonies were subcultured and stored for further use. Subcultured isolates were administered to tests of morphological, biochemical and physiological parameters. Morphological characterization includes size, shape, color, texture, elevation was noted from plates. Bile salt tolerance, acid tolerance, $\mathrm{NaCl}$ tolerance, temperature studies were done under physiological parameters and catalase, oxidase, urease, nitrogen reductase, carbohydrate utilization, MR-VP, citrate utilization tests under biochemical parameters were conducted. Finally promising isolate was subjected to molecular characterization.

\section{Biochemical Characterization:}

Four groups of tests were done

(i) Growth, motility and Gram staining

(ii) Indole-Methyl red Voges-Proskauer citrate (IMVIC) test

(iii) Respiration test (catalase, oxidase, nitrate, urease) test

(iv) Carbohydrate fermentation

\section{Growth, Motility test, and Gram staining:}

Growth and motility of isolates were performed by inoculating on MRS agar plates and incubated the plates at different temperatures for 24-48hours. Temperature includes $4{ }^{0} \mathrm{C}$, $15^{\circ} \mathrm{C}, 25^{\circ} \mathrm{C}, 37^{\circ} \mathrm{C}, 45^{\circ} \mathrm{C}$, and $50^{\circ} \mathrm{C}$. And motility of the isolates was observed by the U-tube method. In this method, U-tube was substituted with MRS semi-solid agar $(0.8 \%)$ and our isolates were inoculated on one side of the U-tube and kept in an upright position, and 
incubated at $37^{\circ} \mathrm{C}$ overnight. If our isolates have motility, turbidity was observed on the other side of the U-tube. To identify whether gram-positive or gram-negative staining technique was performed following Berger's manual for the procedure.

\section{Indole-Methyl red Voges- Proskauer- citrate test:}

This test was performed in four stages with different reagents and dyes. (a) Isolated strains were inoculated in tryptone broth and addition of Kovac's reagent and incubated overnight at $37^{\circ} \mathrm{C}$, the cherry-red color was observed in the broth due to indole production. (b) In methyl red test glucose phosphate media broth was used, isolates were inoculated to the broth and incubated overnight and then methyl red was added to the growing bacteria if the red color disappears it indicates a positive result. (c) Isolates were inoculated in glucose phosphate media broth and incubated at $37^{\circ} \mathrm{C}$ overnight and next day to the growth bacteria VogesProskauer reagent $(40 \% \mathrm{KOH})$ and reagent $11(\alpha$-naphthol) was added, red color ring in test tube indicates the positive result. (d) For Citrate utilization, in Simmons citrate medium culture was inoculated and incubated overnight change of color from green to blue color resembles positive results.

\section{Respiration test: (Catalase, Nitrate, Oxidase):}

Catalase test was performed by addition of $3 \%$ hydrogen peroxide to the loop of isolates on the slide, bubble formation after 1 minute indicates positive test. For nitrate test, isolates were inoculated in nitrate broth and incubated overnight.After incubation sulfanilic acid and $\alpha-$ naphthylamine was added.If the colour of medium changes to red it indicates a positive result. Oxidase test was performed by rubbing the inoculated isolate on to the sterile filter paper strip which was dipped and dried in the fresh tetramethyl-para-phenyl diaminodihydrochloride (oxidase reagent).Violet colour indicates the positive result.

\section{Carbohydrate fermentation:}

All the subcultured isolates were inoculated in the nutrient broth of $\mathrm{pH} 7$ with $1 \%$ carbohydrate, $0.1 \%$ Andrade's indicator in Durham's tubes. $1 \%$ of carbohydrate used in present wok include, arabinose, cellobiose, dextrose, galactose, lactose, mannitol, maltose, melibiose, mannose, ribose, salicin, sorbitol, trehalose, xylose Tubes were incubated at $37^{\circ} \mathrm{C}$ at the anaerobic condition for 24hours. After incubation time bubbles appearance in the Durham's tube indicates the production of gas and colourless to pink/red indicates fermentation.

\section{Survival under GIT conditions: Acid tolerance:}

For acid tolerance assay, MRS broth was prepared with different $\mathrm{pH}$ varying from 2, 3, 4, 5, 6,7 and 8 by using concentrated $\mathrm{HCl}$ and $0.1 \mathrm{~N} \mathrm{NaOH}$. $100 \mu \mathrm{l}$ of the overnight culture was inoculated in broth and incubated in anaerobic conditions. At regular intervals of time 0 , $30 \mathrm{~min}$, 60min, 90min, $120 \mathrm{~min}$, about $100 \mu \mathrm{l}$ isolates were collected.Acid resistance was evaluated by using the plate count method on MRS agar (12).

\section{Bile tolerance:}

Bile tolerance of potential isolates that survived for $3 \mathrm{hrs}$ in acidic condition was determined using MRS broth with $0.3 \%, 0.5 \%$ and $1.0 \%$ of bile. $100 \mu \mathrm{l}$ of the overnight culture was inoculated in broth and incubated in anaerobic conditions. At regular intervals of time 0 , $30 \mathrm{~min}$, 60min, 90min, $120 \mathrm{~min}$, about $100 \mu \mathrm{l}$ isolates were collected.Bile resistance was evaluated by using the plate count method on MRS agar (12). 


\section{Resistance to $0.4 \%$ phenol:}

Phenol tolerance was performed by the addition of $0.4 \%$ phenol to MRS broth, isolates were inoculated and incubated for 24 hours at $37^{\circ} \mathrm{C}$. After incubation time the ability of bacteria to grow in phenol was determined by growth and turbidity of the bacteria (13).

\section{NaCl tolerance:}

$100 \mu 1$ of the overnight culture was inoculated in MRS broth which was adjusted with different salt concentration i.e. $(3 \%, 6 \%, 9 \%$, and $10 \%)$ and incubated at $37^{\circ} \mathrm{C}$ for 24 hours. After incubation growth and turbidity were observed under the spectrophotometer at $600 \mathrm{~nm}$ against the blank (14).

\section{Antibiotic assay:}

The resistance or susceptibility of antibiotics to bacteria was performed by disc diffusion method. Antibiotics used to study are Ampicillin (10mg), Chromophenicol (30mg), Ciproflaxin (10mg), Erythromycin (15mg), Kanamycin (30mg), Penicillin (10 units), Streptomycin (10mg), Tetracycline (30mg), and Vancomycin (10mg) (HiMedia, Mumbai, India).Protocol was followed according to the guidelines of Clinical and Laboratory Standard Institute (CLSI) (15). The overnight culture was swabbed on Muller Hinton media and antibiotic discswere placed on the media and plates were incubated at $37^{\circ} \mathrm{C}$ for 24 hours. After the incubation period, the zone of inhibition was measured.

\section{Antimicrobial assay:}

One of the important criteria for the selection of probiotics is the antimicrobial activity and this assay was performed as per the Agar well diffusion method (16). Escherichia coli, Salmonella species, Staphylococcus aureus, Pseudomonas aeruginosa, Bacillus subtilis, Serratia marcescens, Proteus vulgaris gram-positive and gram-negative bacteria are used for the present study. Actively grown culture was centrifuged at $10,000 \times \mathrm{xg}$ for $20 \mathrm{~min}$ and supernatant was collected and concentrated on a rotary evaporator until one-fifth of the original volume later it was filtered through $0.2 \mu \mathrm{m}$ membrane filter. Pathogen culture was grown separately on nutrient broth at $37^{\circ} \mathrm{C}$ for 24 hours, was included with sterilized nutrient agar $(2.5 \%)$ at $45^{\circ} \mathrm{C}$, now poured in a sterile plate and let it to solidify. After solidification, a well of $0.5 \mathrm{~mm}$ was bored in the plate and filled with filtrate of probiotic isolate and plates were incubated at $37^{\circ} \mathrm{C}$ for 24 hours. After incubation zone of inhibition was measured.

\section{Molecular characterization:}

Molecular identification was utilized to identify the obtained strains. The total Genomic DNA of isolates was extracted using the Genomic DNA purification kit. The primers used for amplifying the $16 \mathrm{~S}$ rRNA sequences are forward 500-AGAGTT TGATCC TGG CTC AG300 and reverse 500-CCGTCA ATT CCT TTGAGT TT-3" (17). The fragments were amplified in an Eppendorf under the following conditions: 94 _C, 30 cycles of 94 _C for $45 \mathrm{~s}$, 55 _C for $30 \mathrm{~s}$ and finally 72 _C for $10 \mathrm{~min}$. The amplified fragment was screened on an agarose gel and sequenced by the Bioport Delhi. Sequences were screened via the BLAST program (https:// blast.ncbi.nlm.nih.gov/Blast.cgi). The sequences were deposited into Gene Bank. Sequence alignment was performed via ClustalW2 (http://www.ebi.ac.uk/Tool/mas/clustalw2/) and a phylogenetic tree was constructed via neighbor-joining (18) and maximum-composite likelihood methods (19) using Mega 6.0 software (http://megasoftware.net/). 


\section{RESULTS:}

\section{Isolation and Identification of Lactobacilli:}

Twenty-seven LAB isolates were isolated from human breast milk, out of which 9 isolates showed probiotic features. Based on all the morphological, Biochemical and probiotic properties, MPS-16 was found abundantly in MRS agar media supplemented with $1 \%$ $\mathrm{CaCO} 3$ and maintained at $4{ }^{\circ} \mathrm{C}$ for further studies. MPS-16 was gram-positive, catalasenegative, rod-shaped, morphologically circular shape, small size, rough surface, moist texture, creamy color, flat elevation, entire margin. Biochemically traits include positive for urease, oxidase, starch hydrolysis, and indole test, whereas negative for catalase, nitrate reduction, methyl red test, Vogues Proskauer test, and citrate utilization. In gas production, carbohydrate fermentation except for raffinose all the sugars showed a positive result. Table 1 clearly shows the result. A phylogenetic tree was constructed using the sequence of MPS-16 (16SrRNA sequence) and a representative sequence from the databases. Phylogenetic analysis utilizing a neighbor-joining dendrogram showed the results that MPS-16 belongs to Lactobacillus rhamnosus (Table-1, 2, Figure 1, and 2).

Table:1 Morphological and biochemical characterizations of the probiotic isolate MPS-16.

\begin{tabular}{|c|c|c|c|c|c|c|c|}
\hline \multicolumn{2}{|c|}{ Morphological trait } & \multicolumn{2}{|l|}{ Biochemical trait } & \multicolumn{4}{|c|}{ Carbohydrate Utilization } \\
\hline Form & circular & Urease test & + & $\begin{array}{l}\text { Gas } \\
\text { production }\end{array}$ & + & Melibiose & + \\
\hline Size & small & Catalase & - & Arabinose & + & Mannose & + \\
\hline Surface & rough & Oxidase & + & Cellobiose & + & Raffinose & - \\
\hline Texture & moist & Nitrate reduction & - & Dextrose & + & Ribose & + \\
\hline Color & creamy & Indole & + & Galactose & + & Salicin & + \\
\hline Elevation & flat & Methyl red test & - & Lactose & + & Sorbitol & + \\
\hline Margin & entire & Vogues Proskauer test & - & Mannitol & + & Trehalose & + \\
\hline $\begin{array}{l}\text { Gram } \\
\text { staining }\end{array}$ & gram +ve cocci & Citrate Utilization & - & Maltose & + & Xylose & + \\
\hline
\end{tabular}

Figure 1: gDNA

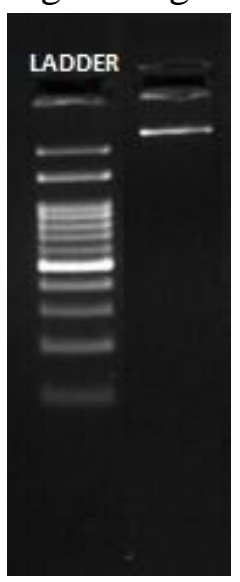

16SrDNA amplicon

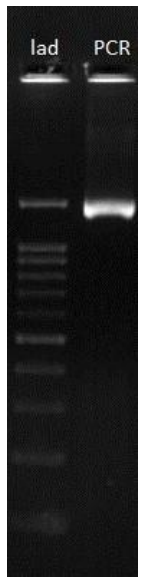

Ladderspecification

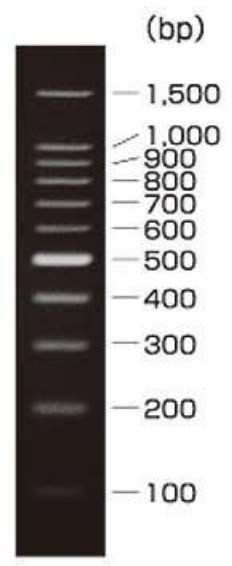


Table2: Sequences producing significant alignments:

\begin{tabular}{|l|c|c|c|c|c|c|}
\hline Description & $\begin{array}{c}\text { Max } \\
\text { Score }\end{array}$ & $\begin{array}{c}\text { Total } \\
\text { score }\end{array}$ & $\begin{array}{l}\text { Query } \\
\text { cover }\end{array}$ & E value & identfication & Accession \\
\hline Lactobacillus rhamnosus strain NBRC 3425 & 2761 & 2761 & $98 \%$ & 0 & $100.00 \%$ & NR_113332.1 \\
\hline Lactobacillus rhamnosus strain JCM 1136 & 2745 & 2745 & $98 \%$ & 0 & $99.67 \%$ & NR_043408.1 \\
\hline Lactobacillus zeae strain RIA 482 & 2699 & 2699 & $98 \%$ & 0 & $99.07 \%$ & NR_037122.1 \\
\hline Lactobacillus casei subsp. casei ATCC 393 & 2695 & 2695 & $99 \%$ & 0 & $98.81 \%$ & NR_041893.1 \\
\hline Lactobacillus casei strain BCRC10697 & 2682 & 2682 & $100 \%$ & 0 & $98.43 \%$ & NR_115322.1 \\
\hline Lactobacillus paracasei strain R094 & 2680 & 2680 & $98 \%$ & 0 & $98.87 \%$ & NR_025880.1 \\
\hline $\begin{array}{l}\text { Lactobacillus casei ATCC 393 strain JCM } \\
1134\end{array}$ & 2673 & 2673 & $98 \%$ & 0 & $98.74 \%$ & NR_115534.1 \\
\hline Lactobacillus paracasei strain NBRC 15889 & 2669 & 2669 & $98 \%$ & 0 & $98.86 \%$ & NR_113337.1 \\
\hline Lactobacillus casei strain NBRC 15883 & 2665 & 2665 & $98 \%$ & 0 & $98.80 \%$ & NR_113333.1 \\
\hline Lactobacillus paracasei strain NBRC 15906 & 2656 & 2656 & $98 \%$ & 0 & $98.73 \%$ & NR_041054.1 \\
\hline
\end{tabular}

\section{PHYLOGENETIC TREE:}

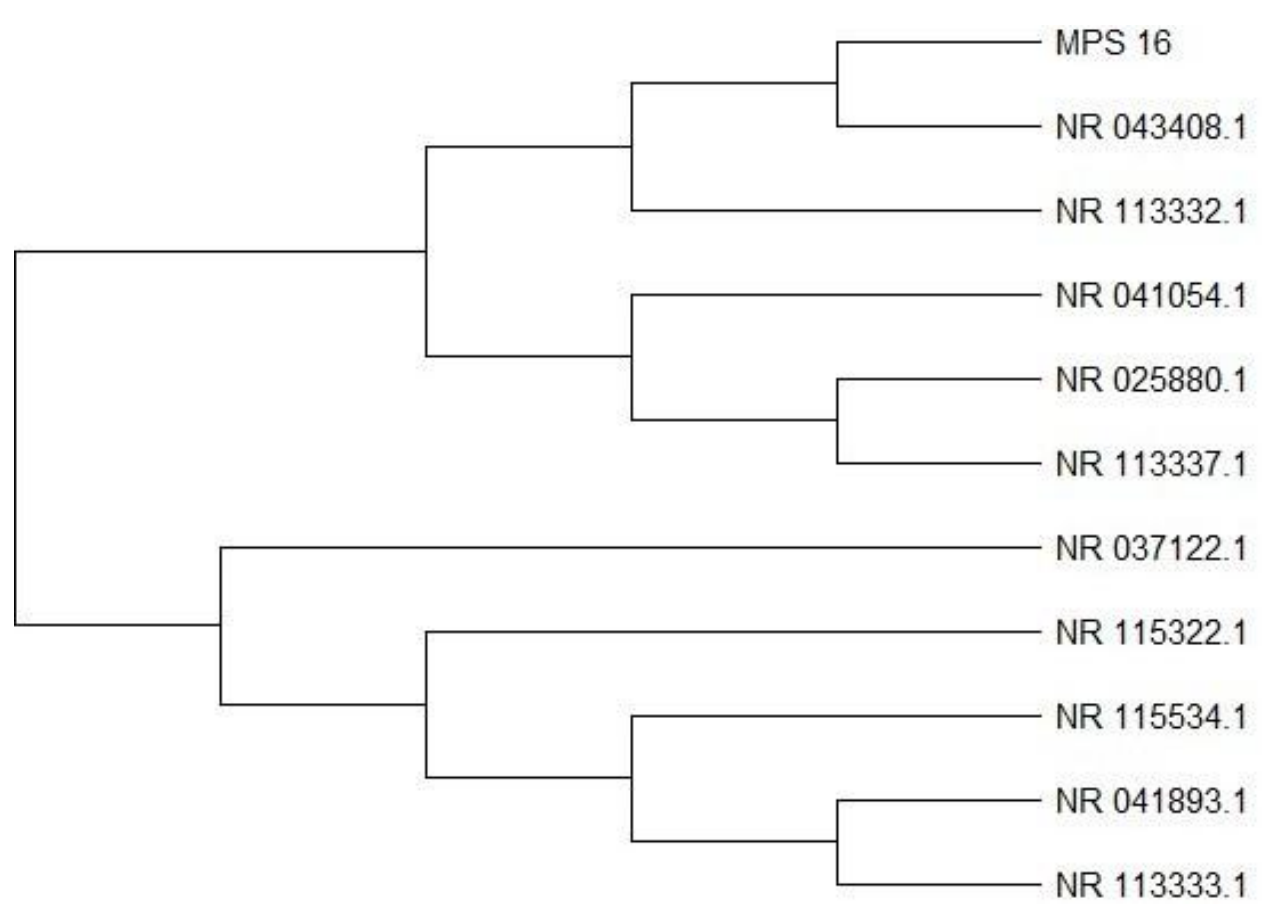

\section{Survival under GIT conditions of MPS-16:}

Acid Tolerance of MPS-16: When MPS-16 was subjected to acid tolerance assay it was found that the ability to survive under gut $\mathrm{pH}$ conditions $\mathrm{pH}-2.0,3.0,4.0$ and 6.5 (control) and resistance or susceptibility was identified by plate count method. Results indicate that MPS-16 has high survival capability in $\mathrm{pH} 2$ and $\mathrm{pH} 3$ shows resistance to acidic conditions (Table 3).

Bile Tolerance of MPS-16: Results suggest that MPS-16 has resistance to various concentrations of bile salts $(0.3 \%, 0.5 \%$, and $1 \%)$. However, an increase of bile concentration beyond this leads to a decrease in the growth and viability of the culture (Table 3 ).

NaCl and Phenol tolerance: MPS-16 shows impactful tolerance to both phenol (0.4) and $\mathrm{NaCl}(3,6,9$, and 12\%) after the incubation period. 
Table 3: Probiotic properties, identity, and NCBI accession number isolate MPS-16

\begin{tabular}{|l|l|l|l|}
\hline \multicolumn{2}{|l|}{ Probiotic properties } & Identification by 16S rDNA analysis & NCBI accession number \\
\hline Acid tolerance $(\mathrm{pH})$ & 2 & Lactobacillus rhamnosus & MN559528 \\
\hline Bile tolerance $(\%)$ & 0.5 & & \\
\hline Phenol tolerance $(\%)$ & 0.4 & & \\
\hline $\mathrm{NaCl}$ tolerance $(\%)$ & 9 & & \\
\hline
\end{tabular}

\section{Antimicrobial and Antibiotic Assay MPS-16:}

Selected lactobacilli MPS-16 was found resistant to tetracycline, streptomycin, kanamycin, chloramphenicol, ciprofloxacin, ampicillin, penicillin, and vancomycin. MPS-16 has also showna clear zone of inhibition against human pathogens including Escherichia coli, Salmonella species, Staphylococcus aureus, Pseudomonas aeruginosa, Bacillus subtilis, Serratia marcescens, Proteus vulgaris. The maximum zone was observed in Serratia, E.coli and Pseudomonas (Table-4).

Table-4: Antibiotic resistance pattern and antimicrobial activity of the probiotic isolate MPS16.

\begin{tabular}{|l|l|l|l|}
\hline \multicolumn{3}{|l|}{ Antibiotic assay zone of inhibition (mm) } & \multicolumn{3}{|l|}{ Antimicrobial assay zone of inhibition(mm) } \\
\hline Ampicillin (10mg) & 24 & Escherichia coli & 18 \\
\hline Chromophenicol (30mg) & 20 & Staphylococcus aureus & 11 \\
\hline Ciprofloxacin (10mg) & 25 & Salmonella species & 14 \\
\hline Erythromycin (15mg) & 24 & Pseudomonas aeruginosa & 16 \\
\hline Kanamycin (30mg) & 17 & Bacillus subtilis & 17 \\
\hline Penicillin (10 units) & 22 & Serratia marcescens & 20 \\
\hline Streptomycin (10mg) & 24 & Proteus vulgaris & 16 \\
\hline Tetracycline (30mg) & 30 & & \\
\hline Vancomycin (10mg) & 29 & & \\
\hline
\end{tabular}

\section{DISCUSSION:}

Breast milk is the best source and important for growth, immunologically, metabolome and microbiome (20). For infants, it is the most important source of gut microflora as it is the only food for newborn babies (21). Research studies suggest that a decrease of lactic acid bacteria in the gut is the major problem for the reduction of immunity and metabolic disorders in infants, adults and old people (22). For this reason, lactic acid bacteria from breast milk are suggested for the maintenance of human health. In our investigation to verify this problem we isolated probiotics from breast milk samples and screened for different conditions similar to the gut condition. Up to now many lactic acid bacteria have been isolated and identified as probiotic bacteria for the development of human and animal health (23). Lactobacillus rhamnosus was identified and concluded that having multiple functions in the supporting of human health, such as protection against pathogenic agents, development, and modulation of immunity (24). May researchers are isolating new lactic acid bacteria strains with high potential capability compared to the previous strains, mainly under gut condition survival rate i.e. low $\mathrm{pH}$ and high bile tolerance which are most important in GIT ( $25,26)$.

In the present study, we isolated 27 strains from human milk out of which MPS-16 showed maximum survival capacity under gut condition, $90 \%$ survival rate at low $\mathrm{pH}$ and high bile salt condition. As per the safety criteria, our isolates showed a maximum zone of inhibition in the case of antibiotic resistance streptomycin, ampicillin, gentamicin, kanamycin, penicillin, 
cephalexin, and ciprofloxacin. These results are similar and consistent with previous results which are attained using L.rhamnosus GG species (27, 28). Antimicrobial assay, i.e production of antimicrobial compounds is one of the main criteria for the selection of probiotic isolate, to inhibit the activity of pathogen and survive under gut condition (29). MPS-16showed maximum zone of inhibition against all pathogenic agents and is consistent with the previous results concluded by Tulumoglu (30).

\section{CONCLUSION:}

Lactobacillus rhamnosus ( $\mathrm{Lr}$ ) was isolated from mother breast milk and indicated as MPS16. Isolate has satisfied all probiotic criteria's and is a non-pathogenic strain. MPS-16 showed maximum survival rate under low $\mathrm{pH}$ and high bile salt conditions, which is essential to survive in GIT conditions. This identified Lactobacillus rhamnosus supports research on gut microbes having novel inter-organ communication and immune development in human metabolic functions.

\section{Conflicts of interest:}

All authors have none to declare.

\section{Acknowledgments:}

We would like to acknowledge and thankful Acharya Nagarjuna University, Department of Biotechnology for the use of their library facilities during the research findings.

\section{REFERENCE:}

1. Food and Agriculture Organization of the United Nations. Document reference. FAO. FAOSTAT. Food and Agriculture Organization of the United Nations. , 2006

2. García-Ruiz, A., de Llano, D. G., Esteban-Fernandez, A., Requena, T., Bartolome, B., (2014). Assessment of probiotic properties in lactic acid bacteria isolated from wine. Food Microbiology, 44, pp.220-225

3. de Vrese, M., \& Schrezenmeir, J. (2008). Probiotics, prebiotics, and synbiotics. Advances in Biochemical Engineering, Biotechnology, 111(8), pp.1-66.

4. Ohland, C. L., and MacNaughton, W. K. (2010). Probiotic bacteria and intestinal epithelial barrier function. American Journal of Physiology Gastrointestinal and Liver Physiology, 298(6), pp.807-819

5. Vidhyasagar, V., and Jeevaratnam, K. (2013). Evaluation of Pediococcus-pentosaceus strains isolated from Idly batter for probiotic properties in vitro. Journal of Functional Foods, 5(1), pp.235-243.

6. Fathabad, E.G., Eslamifar, M. (2011). Isolation and applications of one strain of Lactobacillus paraplantarum from tea leaves (Camellia sinensis). American Journal of Food Technology.,6, pp. 429-434.

7. Erkkila, S., \& Petaja, E. (2000). Screening of commercial meat starter cultures at low pH and in the presence of bile salts for potential probiotic use. Meat Science, 55(3), pp. 297 300.

8. Rubio, R., Jofre, A., Martín, B., Aymerich, T., and Garriga, M. (2014). Characterization of lactic acid bacteria isolated from infant faeces as potential probiotic starter cultures for fermented sausages. Food Microbiology, 38, pp.303-311

9. Kozak, K., Charbonneau, D., Sanozky-Dawes, R., and Klaenhammer, T. (2015). Characterization of bacterial isolates from the microbiota of mothers' breast milk and their infants. Gut Microbes, 6(6), pp. 341-351

10. Martín, R., Langa, S., Reviriego, C., Jimínez, E., Marín, M. L., Xaus, J., and Rodríguez, J. M. (2003). Human milk is a source of lactic acid bacteria for the infant gut. The Journal of Pediatrics, 143(6), pp.754-758. 
11. Rodríguez, E., Arques, J. L., Rodríguez, R., Peiroten, A., Landete, J. M., and Medina, M. (2012). Antimicrobial properties of probiotic strains isolated from breast-fed infants. Journal of Functional Foods, 4(2), pp. 542-551.

12. Oh, Y. J., and Jung, D. S. (2015). Evaluation of probiotic properties of Lactobacillus and Pediococcus strains isolated from Omegisool, a traditionally fermented millet alcoholic beverage in Korea. LWT-food Science and Technology, 63(1), pp. 437-444.

13. Teply M (1984). Cistemlekarskekultury. Phara. SNTL Nakladatelstvi. Technicke Litertury. In: Starters for Fermented Milks, Kurmann JA (Ed), IDF Bulletin .227, pp.4155.

14. Graciela FVD, Maria PT. Food microbiology protocols probiotic properties of Lactobacilli.

15. Wikler, M.A. (2006). Performance standards for antimicrobial susceptibility testing: Sixteenth informational supplement. Clinical and Laboratory Standards Institute, Wayne, PA.

16. Aween, M.M., Hassan, Z., Muhialdin, B.J., Eljamel, Y.A., Mabrok, A.S.W.A., and Lani, M.L.(2012). Antibacterial activity of Lactobacillus acidophilus strains isolated from honey marketed in Malaysia against selected multiple antibiotic resistant (MAR) gram positive bacteria. Journal of Food Science, 77, pp. 364-371

17. Beasley, S. S., and Saris, P. E. (2004). Nisin-producing Lactococcus-lactis strains isolated from human milk. Applied and Environmental Microbiology, 70(8), pp.50515053.

18. Saitou, N., and Nei, M. (1987). The neighbor-joining method: A new method for reconstructing phylogenetic trees. Molecular Biology and Evolution, 4(4), pp. 406-425

19. Tamura, K., Nei, M., and Kumar, S. (2004). Prospects for inferring very large phylogenies by using the neighbor joining method. Proceedings of the National Academy of Sciences of the United States of America, 101(30), pp. 11030-11035.

20. Aaltonen, J., Ojala, T., Laitinen, K., Poussa, T., Ozanne, S., and Isolauri, E. (2011). Impact of maternal diet during pregnancy and breastfeeding on infant metabolic programming: A prospective randomized controlled study. European Journal of Clinical Nutrition, 65(1), pp.10-19.

21. Arici, M., Bilgin, B., Sagdic, O., and Ozdemir, C. (2004). Some characteristics of Lactobacillus isolates from infant-faeces. Food Microbiology, 21(1),pp. 19-24

22. Tsai, Y.T., Cheng, P.C., \& Pan, T.M. (2012). The immunomodulatory effects of lactic acid bacteria for improving immune functions and benefits. Applied Microbiology and Biotechnology, 96(4), pp. 853-862.

23. Rajoka, M. S. R., Shi, J., Zhu, J., Shao, D., Huang, Q., and Yang, H. (2017). Capacity of lactic acid bacteria in immunity enhancement and cancer prevention. Applied Microbiology and Biotechnology, 101(1), pp. 35-45.

24. Kaewiad, K., Kaewnopparat, S., and Kaewnopparat, N. (2015). In vitro comparison of probiotic properties of Lactobacillus fermentum SK54 isolated from new born baby with Lactobacillus rhamnosus GG ATCC 53103. Advanced Materials Research, 1060, pp. 215-218.

25. Mandal, H., Jariwala, R., and Bagchi, T. (2015). Isolation and characterization of lactobacilli from human faeces and indigenous fermented foods for their potential application as probiotics. Canadian Journal of Microbiology, 62(4), pp.349-359.

26. Oh, Y. J., and Jung, D. S. (2015). Evaluation of probiotic properties of Lactobacillus and Pediococcus strains isolated from Omegisool, a traditionally fermented millet alcoholic beverage in Korea. LWT-food Science and Technology, 63(1), pp. 437-444. 
27. Chang, L., Zhang, Z.-Y., Ke, D., Jian-Ping, Y., and Xiao-Kui, G. (2009). Antibiotic resistance of probiotic strains of lactic acid bacteria isolated from marketed foods and drugs. Biomedical and Environmental Sciences, 22(5), pp. 401-412.

28. Maragkoudakis, P. A., Zoumpopoulou, G., Miaris, C., Kalantzopoulos, G., Pot, B., and Tsakalidou, E. (2006). Probiotic potential of Lactobacillus strains isolated from dairy products. International Dairy Journal, 16(3), 189e199.

29. Collado, M. C., Gueimonde, M., Sanz, Y., \& Salminen, S. (2005). Adhesion of selected Bifidobacterium-strains to human intestinal mucus and the role of adhesion in enteropathogen exclusion. Journal of Food Protection, 68(12), pp. 2672-2678.

30. Tulumoglu, S., Yuksekdag, Z. N., Beyatli, Y., Simsek, O., Cinar, B., \& Yas, ar, E. (2013). Probiotic properties of lactobacilli species isolated from children's feces. Anaerobe, 24, pp.36-42. 